\title{
A New Monte Carlo Approach for Conservation Laws and Relaxation Systems
}

\author{
Lorenzo Pareschi ${ }^{1}$ and Mohammed Seaïd ${ }^{2}$ \\ 1 Department of Mathematics, University of Ferrara, 44100 Italy \\ pareschi@dm.unife.it \\ 2 Fachbereich Mathematik AG8, TU Darmstadt, 64289 Darmstadt, Germany \\ seaid@mathematik.tu-darmstadt.de
}

\begin{abstract}
We present a Monte Carlo method for approximating the solution of conservation laws. A relaxation method is used to transform the conservation law to a kinetic form that can be interpreted in a probabilistic manner. A Monte Carlo algorithm is then used to simulate the kinetic approximation. The method we present in this paper is simple to formulate and to implement, and can be straightforwardly extended to higher dimensional conservation laws. Numerical experiments are carried out using Burgers equation subject to both smooth and nonsmooth initial data.
\end{abstract}

\section{Introduction}

Monte Carlo methods have been always very popular in scientific computing. This is mainly due to the ability to deal efficiently with very large (multiscale) structures without many meshing problems and to their simplicity in keeping the fundamental physical properties of the problems. In particular Monte Carlo methods have been widely used for numerical simulations in rarefied gas dynamics described by the Boltzmann equation 15. More recently these methods have been extended to treat regimes close to continuum situations described by the Euler or Navier-Stokes equations 78,92 . The common idea in these approximations is to take advantage of the knowledge of the equilibrium state of the equation to build a scheme with the correct behavior close to the fluid-limit. For example, for the Boltzmann equation close to fluid regimes particles are sampled directly from a Maxwellian distribution as in Pullin's method 10. In this article, inspired by these methods, we use a relaxation approximation to transform a conservation law into a semilinear system which has the structure of a discrete velocity model of the Boltzmann equation. This kinetic form leads naturally to a probabilistic representation. Therefore, the main ideas used in [789] can be used to simulate the limiting conservation law. More precisely advection of particles is made according to the characteristic speeds of the relaxation system, and the projection into the equilibrium is performed with a suitable sampling strategy.

Let consider the scalar conservation law

$$
\begin{aligned}
& u_{t}+\varphi(u)_{x}=0, \quad(x, t) \in \mathbb{R} \times \mathbb{R}^{+}, \\
& u(x, t=0)=u_{0}(x), \quad x \in \mathbb{R},
\end{aligned}
$$


where $u \in \mathbb{R}$ and the flux function $\varphi(u): \mathbb{R} \rightarrow \mathbb{R}$ is nonlinear. As in 3], we replace the scalar Cauchy problem (1) by the semilinear relaxation system

$$
\begin{gathered}
u_{t}+v_{x}=0, \\
v_{t}+a u_{x}=-\frac{1}{\varepsilon}(v-\varphi(u)), \\
u(x, 0)=u_{0}(x), \quad v(x, 0)=\varphi\left(u_{0}(x)\right),
\end{gathered}
$$

where $v \in \mathbb{R}, a$ is positive constant, and $\varepsilon$ is the relaxation rate. When $\varepsilon \longrightarrow 0$, solution of the relaxation system (2) approaches solution of the original equation (1) by the local equilibrium $v=\varphi(u)$. A necessary condition for such convergence is that the subcharacteristic condition 36 .

$$
-a \leq \varphi^{\prime}(u) \leq a, \quad \forall u,
$$

is satisfied in (2). The main advantage in considering the relaxation method lies essentially on the semilinear structure of the system (2), which has two linear characteristic variables (given by $v \pm \sqrt{a} u$ ), and consequently it can be solved numerically without using Riemann solvers (see 3 and the references therein).

Our purpose in the present paper is to construct a Monte Carlo approach for the conservation law (11) using the fact that, for the relaxation system (2) a kinetic formulation can be easily derived. The organization of the paper is as follows. Section 2 is devoted to the probabilistic formulation for the relaxation system (2). In section 3 we discuss a Monte Carlo algorithm for the relaxation model. Section 4 illustrates the performance of the approach through experiments with the Burgers equation. In the last section some concluding remarks and future developments are listed.

\section{Probabilistic Interpretation}

In order to develop a probabilistic formulation for the relaxation system (2) we introduce the kinetic variables $f$ and $g$ as

$$
u=f+g \quad \text { and } \quad v=a(f-g) .
$$

The relaxation system can be rewritten in a diagonal form as

$$
\begin{aligned}
& f_{t}+\sqrt{a} f_{x}=\frac{1}{\varepsilon}\left(\frac{g-f}{2}+\frac{\varphi(f+g)}{2 \sqrt{a}}\right), \\
& g_{t}-\sqrt{a} g_{x}=\frac{1}{\varepsilon}\left(\frac{f-g}{2}-\frac{\varphi(f+g)}{2 \sqrt{a}}\right) .
\end{aligned}
$$

To solve numerically the equations (15) we split the problem into two steps:

(i) The transport stage

$$
\begin{aligned}
& f_{t}+\sqrt{a} f_{x}=0, \\
& g_{t}-\sqrt{a} g_{x}=0 .
\end{aligned}
$$


(ii) The relaxation stage

$$
\begin{aligned}
& f_{t}=\frac{1}{\varepsilon}\left(\frac{g-f}{2}+\frac{\varphi(f+g)}{2 \sqrt{a}}\right), \\
& g_{t}=\frac{1}{\varepsilon}\left(\frac{f-g}{2}-\frac{\varphi(f+g)}{2 \sqrt{a}}\right) .
\end{aligned}
$$

Note that this splitting is first order accurate. A second order splitting for moderately stiff relaxation stages can be derived analogously using the Strang method [1]. For simplicity first we will describe the relaxation problem (7), and then we show how to combine it with the stage (6) for the full problem.

We assume, without loss of generality, that for a fixed $x$

$$
f \geq 0, \quad g \geq 0, \quad u=f+g=1 .
$$

Furthermore, we assume that the flux function in (1) satisfies

$$
\varphi^{\prime}(u)<1, \quad 0 \leq \varphi(u) \leq u .
$$

Although problem (7) can be solved exactly we consider its time discretization

$$
\begin{aligned}
& f^{n+1}=f^{n}+\frac{\Delta t}{\varepsilon}\left(\frac{g^{n+1}-f^{n+1}}{2}+\frac{\varphi\left(f^{n+1}+g^{n+1}\right)}{2}\right), \\
& g^{n+1}=g^{n}+\frac{\Delta t}{\varepsilon}\left(\frac{f^{n+1}-g^{n+1}}{2}-\frac{\varphi\left(f^{n+1}+g^{n+1}\right)}{2}\right) .
\end{aligned}
$$

Since $f^{n+1}+g^{n+1}=f^{n}+g^{n}$ we can write

$$
\begin{aligned}
& f^{n+1}=f^{n}+\frac{\Delta t}{\varepsilon}\left(\frac{\varepsilon}{\varepsilon+\Delta t} \frac{g^{n+1}-f^{n+1}}{2}+\frac{\varepsilon}{\varepsilon+\Delta t} \frac{\varphi\left(f^{n+1}+g^{n+1}\right)}{2}\right), \\
& g^{n+1}=g^{n}+\frac{\Delta t}{\varepsilon}\left(\frac{\varepsilon}{\varepsilon+\Delta t} \frac{f^{n+1}-g^{n+1}}{2}-\frac{\varepsilon}{\varepsilon+\Delta t} \frac{\varphi\left(f^{n+1}+g^{n+1}\right)}{2}\right) .
\end{aligned}
$$

Or equivalently

$$
\begin{aligned}
& f^{n+1}=(1-\lambda) f^{n}+\lambda\left(\frac{g^{n+1}-f^{n+1}}{2}+\frac{\varphi\left(f^{n+1}+g^{n+1}\right)}{2}\right), \\
& g^{n+1}=(1-\lambda) g^{n}+\lambda\left(\frac{f^{n+1}-g^{n+1}}{2}-\frac{\varphi\left(f^{n+1}+g^{n+1}\right)}{2}\right),
\end{aligned}
$$

with $\lambda=\frac{\varepsilon}{\varepsilon+\Delta t}$. Now let us define the probability density

$$
P^{n}(\xi)= \begin{cases}f^{n}, & \text { if } \xi=+\sqrt{a} \\ g^{n}, & \text { if } \xi=-\sqrt{a} \\ 0, & \text { elsewhere. }\end{cases}
$$


Note that $0 \leq P^{n}(\xi) \leq 1$ and $\sum_{\xi} P^{n}(\xi)=1$. Moreover

$$
\sum_{\xi} P^{n+1}(\xi)=\sum_{\xi} P^{n}(\xi)=1 .
$$

The system (10) can be seen as the evolution of the probability function $P^{n}(\xi)$ according with

$$
P^{n+1}(\xi)= \begin{cases}(1-\lambda) f^{n}+\lambda E_{f}\left(u^{n}\right), & \text { if } \xi=+\sqrt{a} \\ (1-\lambda) g^{n}+\lambda E_{g}\left(u^{n}\right), & \text { if } \xi=-\sqrt{a} \\ 0, & \text { elsewhere }\end{cases}
$$

where $E_{f}(u)=\frac{u+\varphi(u)}{2}$ and $E_{g}(u)=\frac{u-\varphi(u)}{2}$.

We remark that, since $\varepsilon>0$, the condition $0 \leq \lambda \leq 1$ is satisfied. Obviously, (11) represents a convex combination of two probability densities. In fact, thanks to (8), we have $E_{f}(u) \geq 0, E_{g}(u) \geq 0$ and $E_{f}(u)+E_{g}(u)=1$.

\section{Monte Carlo Algorithm}

In order to develop a Monte Carlo game to approach the solution to the kinetic system (5), in addition to a good random number generator, we need a way to sample particles from an initial data and some other basic tools which are described with details in the lecture notes [9]. Thus, we consider two families of particles that defines samples of our probability density $P^{n}(\xi)$.

Let us define with $\left\{\xi_{1}, \xi_{2}, \ldots, \xi_{N}\right\}$ the particle samples, we know that $\xi_{j}=$ $+\sqrt{a}$ or $\xi_{j}=-\sqrt{a}$ with probability $f^{n}$ or $g^{n}$ respectively. We have the relation

$$
P^{n+1}(\xi)=(1-\lambda) P^{n}(\xi)+\lambda E^{n}(\xi),
$$

where $E^{n}(\xi)$ is defined as: $E^{n}(\xi)= \begin{cases}\frac{u+\varphi(u)}{2}, & \text { if } \xi=+\sqrt{a}, \\ \frac{u-\varphi(u)}{2}, & \text { if } \xi=-\sqrt{a}, \\ 0, & \text { elsewhere. }\end{cases}$

Hence, the relaxation stage (7) can be solved in the following way:

- Given a particle sample $\xi$ the evolution of the sample during the time integration process is performed according to :

$\circ$ with probability $(1-\lambda)$ the sample is unchanged

o with probability $\lambda$ the sample is replaced with a sample from $E^{n}(\xi)$

- To sample a particle from $E^{n}(\xi)$ we proceed as follows:

○ with probability $\frac{u+\varphi(u)}{2}$ take $\xi=+\sqrt{a}$

○ with probability $\frac{u-\varphi(u)}{2}$ take $\xi=-\sqrt{a}$ 

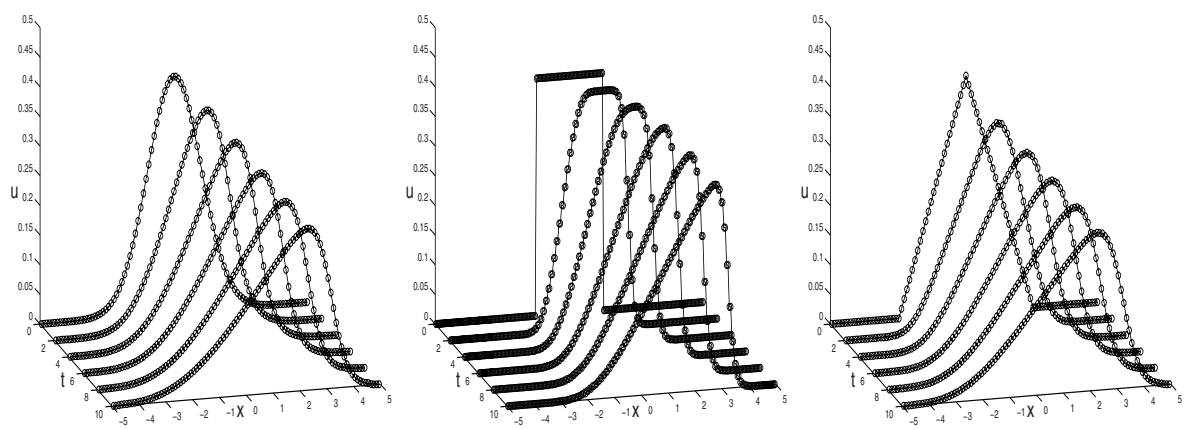

Fig. 1. Evolution in time-space of Gaussian distribution (right), box distribution (medium) and cone distribution (left) using the Monte Carlo approach.

Note that the relaxation stage is well defined for any value of $\epsilon$. In particular as $\epsilon \rightarrow 0$ we have $\lambda \rightarrow 1$ and thus particles are all sampled from the local equilibrium $E^{n}(\xi)$. To generate particles the spatial domain is first divided into cells $\left[x_{i-\frac{1}{2}}, x_{i+\frac{1}{2}}\right]$ with stepsize $\Delta x$ and centered in $x_{i}$. Then particles are generated from a given piecewise initial data in each cell and are randomly distributed around the cell center $x_{i}$.

Once the particle distribution is updated by the above steps, the transport stage (6) of the splitting is realized by advecting the position of the particles according to their speeds. Thus, given a sample of $N$ particles at positions $x_{1}, x_{2}, \cdots, x_{N}$ and speeds $\xi_{1}, \xi_{2}, \cdots, \xi_{N}$ (equal either to $+\sqrt{a}$ or $-\sqrt{a}$ ) the new position of the particle sample $\left\{x_{i}, \xi_{i}\right\}$ is simply

$$
x_{i}^{\text {new }}=x_{i}^{\text {old }}+\Delta t \xi_{i}, \quad i=1, \ldots, N,
$$

where $x_{i}^{\text {new }}$ and $x_{i}^{\text {old }}$ are respectively, the new and old positions of the sample $\xi_{i}$, and $\Delta t$ is the time stepsize.

Remark 1 The Monte Carlo method presented in this paper applies also if $u>0$ and $u \neq 1$. For this case

$$
P^{n}(\xi)=\left\{\begin{array}{ll}
\frac{f^{n}}{u_{n}^{n}}, & \text { if } \xi=+\sqrt{a}, \\
\frac{g^{n}}{u^{n}}, & \text { if } \xi=-\sqrt{a}, \\
0, & \text { elsewhere. }
\end{array} \quad \text { and } \quad E^{n}(\xi)= \begin{cases}\frac{u+\varphi(u)}{2 u}, & \text { if } \xi=+\sqrt{a}, \\
\frac{u-\varphi(u)}{2 u}, & \text { if } \xi=-\sqrt{a}, \\
0, & \text { elsewhere. }\end{cases}\right.
$$

It is easy to verify that in this case, $P^{n}$ defines a probability (i.e., $0 \leq P^{n}(\xi) \leq 1$ and $\left.\sum_{\xi} P^{n}(\xi)=1\right)$.

\section{Numerical Results}

In what follows we present numerical results obtained for some tests on Burgers equation given by the equation (1) with the flux function defined as $\varphi(u)=\frac{u^{2}}{2}$. 
Relaxed Monte Carlo results for the Gaussian distribution (13)
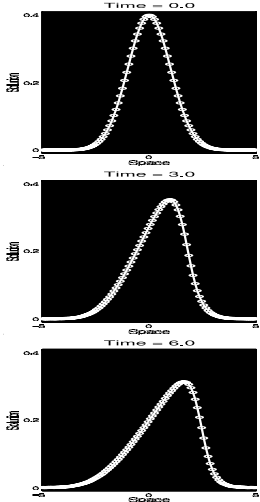

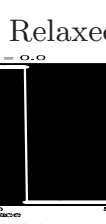
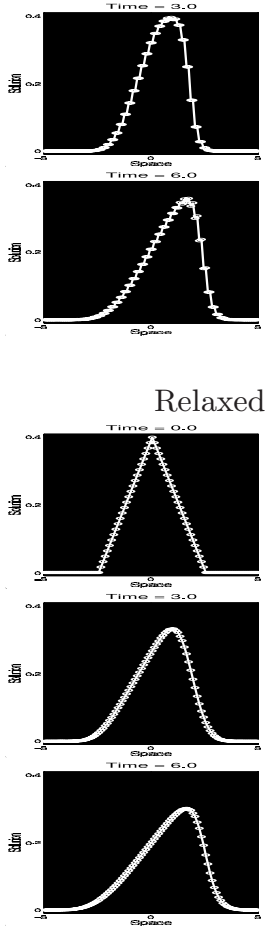
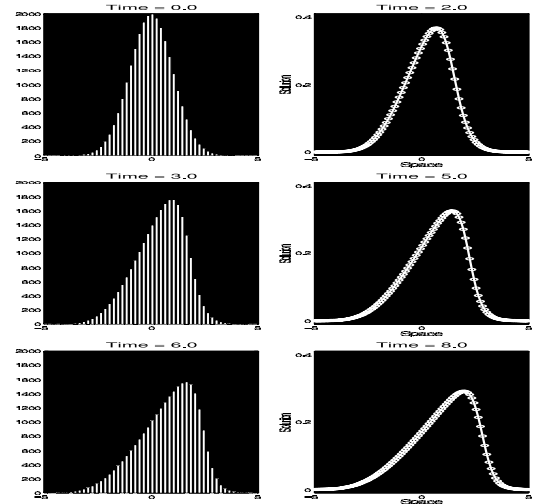
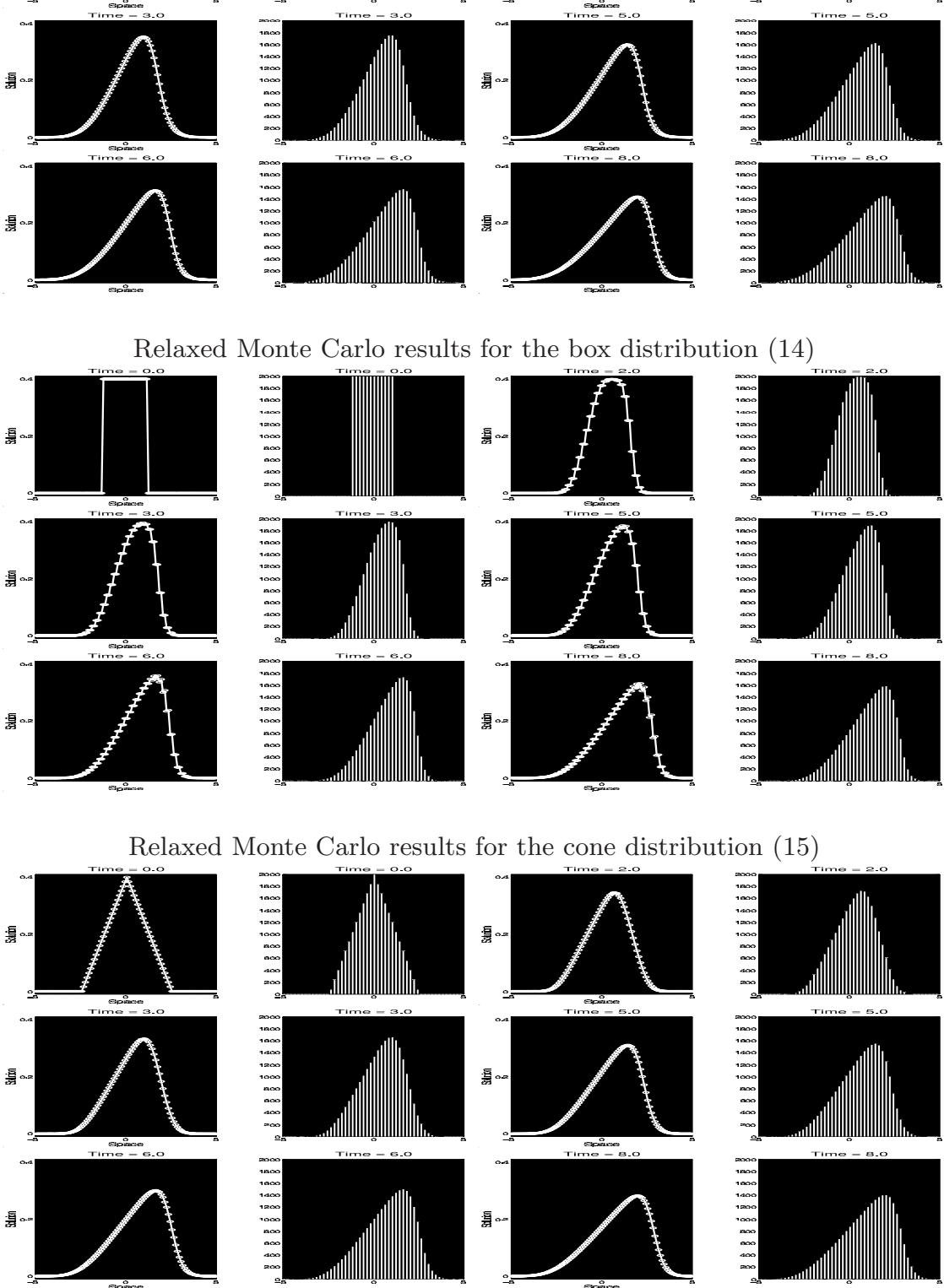

Fig. 2. Relaxed Monte Carlo results for the distributions (13)-15). 
Note that this flux function satisfies the assumptions (8). We solve the Burgers equation in the space interval $[-\delta, \delta]$ subject to periodic boundary conditions and using the following three different initial data:

1. Gaussian distribution

$$
u_{0}(x)=\frac{1}{\sqrt{2 \pi}} e^{-\frac{1}{2} x^{2}}
$$

2. Box distribution

$$
u_{0}(x)= \begin{cases}\frac{2}{\delta}, & \text { if } \quad|x| \leq \frac{\delta}{4} \\ 0, & \text { elsewhere }\end{cases}
$$

3. Cone distribution

$$
u_{0}(x)= \begin{cases}-\frac{4|x|}{\delta^{2}}+\frac{2}{\delta}, & \text { if }|x| \leq \frac{\delta}{2} \\ 0, & \text { elsewhere }\end{cases}
$$

Note that the total mass in these initial data is equal to unity, $\int_{\mathbb{R}} u_{0}(x) d x=1$. In all our computations we used $\delta=5$, the spatial interval is discretized into $M=100$ gridpoints uniformly spaced. The number of particles $N$ is set to $10^{4}$ which is large enough to decrease the effect of the fluctuations in the computed solutions. Here we present only results for the relaxed case (i.e. $\varepsilon=0$ ). Results for the relaxing case $(i . e . \varepsilon \neq 0)$ can be done analogously. The time step $\Delta t$ is chosen such a way the $\mathrm{CFL}=1$ and the computation have been performed with a single run for different time $t \in[0,10]$.

In Fig. 1 we display the evolution in time of these initial distributions. For the sake of comparison, we have included in the figures the results obtained by the Lax-Friedrichs method 4, plotted by the solid lines. In the Monte Carlo approach the solution $u\left(x_{i}, t\right)$ at the gridpoint $x_{i}$ and time $t$ has been reconstructed by averaging the number of particles in each cell as

$$
u\left(x_{i}, t\right)=\frac{n\left(x_{i}, t\right)}{N} \sum_{j=1}^{M} u\left(x_{j}, t=0\right), \quad i=1, \ldots, M,
$$

where $n\left(x_{i}, t\right)$ denotes the number of the particles localized in the cell $\left[x_{i-\frac{1}{2}}, x_{i+\frac{1}{2}}\right]$ at time $t$. As can be seen the shock is well captured by the Monte Carlo method. Fig. 2] shows again the results for the initial data (13)-115) along with the evolution of particle distribution in the space interval for six different times. The Monte Carlo approach preserves the positivity of the solution as well as the conservation of mass

$$
\int_{\mathbb{R}} u(x, t) d x=\int_{\mathbb{R}} u_{0}(x) d x=1, \quad \forall t>0 .
$$

We would like to mention that the new Monte Carlo approach can approximate conservation laws with diffusive source terms, for example viscous Burgers equations. The diffusion stage in the algorithm can be treated, for example, by the well-known Random walk method. 


\section{Concluding Remarks}

We have presented a simple Monte Carlo algorithm for the numerical solution of conservation laws and relaxation systems. The algorithm takes advantage of the relaxation model associated to the equation under consideration which can be regarded as the evolution in time of a probability distribution. Although we have restricted our numerical computations to the case of one-dimensional scalar problems, the most important implication of our research concerns the use of effective Monte Carlo procedures for multi-dimensional systems of conservation laws with relaxation terms similarly to the Broadwell system and the BGK model in rarefied gas dynamics. Our current effort is therefore to extend this approach to systems of conservation laws in higher space dimensions. Another extension will be to couple the Monte Carlo method at the large $\epsilon$ scale with a deterministic method at the reduced small $\epsilon$ scale as in [2] for a general relaxation system. Finally we remark that the Monte Carlo approach proposed in this paper is restricted to first order accuracy. A second order method it is actually under study.

Acknowledgements. The work of the second author was done during a visit at Ferrara university. The author thanks the department of mathematics for the hospitality and for technical and financial support. Support by the European network HYKE, funded by the EC as contract HPRN-CT-2002-00282, is also acknowledged.

\section{References}

1. Bird G.A.: Molecular Gas Dynamics. Oxford University Press, London, (1976)

2. Caflisch R.E., Pareschi L.: An implicit Monte Carlo Method for Rarefied Gas Dynamics I: The Space Homogeneous Case. J. Comp. Physics 154 (1999) 90-116

3. Jin S., Xin, Z.: The Relaxation Schemes for Systems of Conservation Laws in Arbitrary Space Dimensions. Comm. Pure Appl. Math. 48 (1995) 235-276

4. LeVeque Randall J.: Numerical Methods for Conservation Laws. Lectures in Mathematics ETH Zürich, (1992)

5. Nanbu K.: Direct Simulation Scheme Derived from the Boltzmann Equation. J. Phys. Soc. Japan, 49 (1980) 2042-2049

6. Natalini, R.: Convergence to Equilibrium for Relaxation Approximations of Conservation Laws. Comm. Pure Appl. Math. 49 (1996) 795-823

7. Pareschi L., Wennberg B.: A Recursive Monte Carlo Algorithm for the Boltzmann Equation in the Maxwellian Case. Monte Carlo Methods and Applications 7 (2001) 349-357

8. Pareschi L., Russo G.: Time Relaxed Monte Carlo Methods for the Boltzmann Equation. SIAM J. Sci. Comput. 23 (2001) 1253-1273

9. Pareschi L., Russo G.: An Introduction to Monte Carlo Methods for the Boltzmann Equation. ESAIM: Proceedings 10 (2001) 35-75

10. Pullin D.I.: Generation of Normal Variates with Given Sample. J. Statist. Comput. Simulation 9 (1979) 303-309

11. Strang, G.: On the Construction and the Comparison of Difference Schemes. SIAM J. Numer. Anal. 5 (1968) 506-517 